Keywords: prostate cancer; biomarkers; single-nucleotide polymorphisms; biochemical recurrence; castrate metastasis; disease-specific mortality

\title{
An analysis of the association between prostate cancer risk loci, PSA levels, disease aggressiveness and disease-specific mortality
}

J Sullivan ${ }^{\star}, 1,2$, R Kopp ${ }^{1,2}$, K Stratton $^{1,2}$, C Manschreck ${ }^{1}$, M Corines ${ }^{1}$, R Rau-Murthy ${ }^{1}$, J Hayes ${ }^{1}$, A Lincon ${ }^{1}$, A Ashraf ${ }^{1}, \mathrm{~T}_{\text {Thomas }}{ }^{1}$, K Schrader ${ }^{1}$, D Gallagher ${ }^{1}$, R Hamilton $^{1}$, H Scher ${ }^{3}$, H Lilja ${ }^{2}$, P Scardino ${ }^{2}$, J Eastham ${ }^{2}$, K Offit ${ }^{1}$, J Vijai ${ }^{1}$ and R J Klein ${ }^{1,4}$

${ }^{1}$ Department of Medicine, Clinical Genetics Service, Memorial Sloan-Kettering Cancer Center, New York, NY, USA; ${ }^{2}$ Department of Surgery, Urology Service, Memorial Sloan-Kettering Cancer Center, New York, NY, USA; ${ }^{3}$ Department of Medicine, Genitourinary Medical Oncology, Memorial Sloan-Kettering Cancer Center, New York, NY, USA and ${ }^{4}$ Icahn Institute for Genomics and Multiscale Biology, Department of Genetics and Genomic Sciences, Icahn School of Medicine at Mount Sinai, New York, NY, USA

Background: Genome-wide association studies have identified multiple single-nucleotide polymorphsims (SNPs) associated with prostate cancer (PCa). Although these SNPs have been clearly associated with disease risk, their relationship with clinical outcomes is less clear. Our aim was to assess the frequency of known PCa susceptibility alleles within a single institution ascertainment and to correlate risk alleles with disease-specific outcomes.

Methods: We genotyped 1354 individuals treated for localised PCa between June 1988 and December 2007. Blood samples were prospectively collected and de-identified before being genotyped and matched to phenotypic data. We investigated associations between 61 SNPs and disease-specific end points using multivariable analysis and also determined if SNPs were associated with PSA at diagnosis.

Results: Seven SNPs showed associations on multivariable analysis $(P<0.05)$, rs 13385191 with both biochemical recurrence (BR) and castrate metastasis (CM), rs339331 (BR), rs1894292, rs17178655 and rs11067228 (CM), and rs11902236 and rs4857841 PCa-specific mortality. After applying a Bonferroni correction for number of SNPs $(P<0.0008)$, the only persistent significant association was between rs17632542 (KLK3) and PSA levels at diagnosis $\left(P=1.4 \times 10^{-5}\right)$.

Conclusions: We confirmed that rs 17632542 in KLK3 is associated with PSA at diagnosis. No significant association was seen between loci and disease-specific end points when accounting for multiple testing. This provides further evidence that known PCa risk SNPs do not predict likelihood of disease progression.

Although prostate cancer $(\mathrm{PCa})$ is highly prevalent and prostatespecific antigen (PSA) screening has led to an abundant diagnosis of disease, including many indolent cases, a substantial number of men will still develop symptomatic metastases or die from their cancer. The ability to identify individuals with a more aggressive disease phenotype would result in more appropriate initial treatment strategies. The need for novel biomarkers to add predictive capacity to existing clinical nomograms at time of diagnosis is of upmost relevance.

Inherited germline susceptibility loci, such as single-nucleotide polymorphisms (SNPs) have the potential to be effective biomarkers, not only in screening for disease but also in

*Correspondence: Dr J Sullivan; E-mail: johnsullivan@rcsi.ie

Received 4 February 2015; revised 24 April 2015; accepted 5 May 2015; published online 11 June 2015

(c) 2015 Cancer Research UK. All rights reserved 0007-0920/15 
contributing to predicting recurrence and response to specific treatments. Since the first PCa genome-wide association study (GWAS) in 2006, there are now over 75 such SNPs associated with disease risk (Eeles et al, 2013). Although related to risk, there is far less known about the ability of these SNPs to discriminate aggressive disease. To date, there are only a handful of studies that have looked at the association of PCa risk loci and disease-specific end points (Penney et al, 2009; Wiklund et al, 2009; Gallagher et al, 2010; Pomerantz et al, 2011; Szulkin et al, 2012; Shui et al, 2014). Many of these studies have small cohorts, have variability across institutions, and lack granular data on disease extent, treatment and length of follow-up.

In this study, we aimed to assess the frequency of a large selection of validated PCa susceptibility alleles from previously reported GWAS within a single institution ascertainment of $\mathrm{PCa}$ patients with long-term follow-up in order to determine association between risk alleles and disease outcomes, including clinical disease progression and PCa-specific mortality (PCSM).

\section{MATERIALS AND METHODS}

Study population. The study population consisted of 1354 men treated for localised PCa at the Memorial Sloan-Kettering Cancer Center between June 1988 and December 2007. Seven hundred and sixty-two individuals identified themselves as being of Ashkenazi Jewish descent, whereas over $90 \%$ of the remaining 592 were selfreported non-Jewish Caucasian individuals. Blood samples were drawn and medical records were collected as part of an institutional PCa research database using standardised questionnaires and chart abstraction forms. Pertinent clinical data included disease stage (TNM classification), Gleason score (from needle biopsy), PSA levels and age at diagnosis, as well as dates of biochemical recurrence (BR), development of castration-resistant metastasis (CM), PCSM and overall patient survival.

All patient records were reviewed by physicians to confirm the clinical end points being tested. Age at diagnosis was considered as the date of first positive prostate biopsy. BR was defined as a single measure of PSA $\geqslant 0.2 \mathrm{ng} \mathrm{ml}^{-1}$ after radical prostatectomy, and a value of 'nadir +2' after other therapy (Stephenson et al, 2006; Nielsen et al, 2008). CM was defined as time of progression of disease following initiation of antiandrogen therapy. Review of the patient's death certificate and/or medical record identified cause of death. In accordance with an institutional research board-approved protocol, patient identifiers were removed at the time of genetic analysis.

Selection of SNPs and genotyping. A total of 75 susceptibility loci of interest were identified, the majority of which were selected on the basis of being significantly associated with PCa risk from previous published GWAS $(n=67)$, the remainder were SNPs associated with PSA levels $(n=6)$. Established PCa risk SNPs that we previously evaluated for association with disease end points in a subset of this cohort were excluded (Gallagher et al, 2010). SNPs were genotyped using the Mass ARRAY QGE iPLEX system (Sequenom, Inc. San Diego, CA, USA; Gabriel et al, 2009). PCR and extension primers for SNPs were designed over three separate multiplex assays using Mass ARRAY Assay Design 3.0 software (Sequenom, Inc). PCR and extension reactions were performed according to the manufacturer's instructions, and extension product sizes were determined by mass spectrometry using the Sequenom iPLEX system. Duplicate test samples and negative controls were also included. In all, 14 of 75 SNPs (19\%) failed quality control and were removed from the analysis. The remaining 61 SNPs had an average genotype call rate of $86 \%$, with each SNP being in Hardy-Weinberg equilibrium (Tables 1 and 2). The minor allele frequency in the study cohort ranged from 1 to $49 \%$. The average control rate among duplicate samples was $98 \%$.
Statistical methods. Univariate Cox proportional hazards regression was used to investigate the association between each SNP and BR, CM, and PCSM. Each SNP was analysed under an additive model. The risk allele for each PCa SNP was defined as the allele associated with an increased risk of disease in the literature. Time at risk was calculated from the date of diagnosis to the date of event or date of last contact, and patients without the event were censored at their last follow-up date.

Multivariable analyses were conducted controlling for selfreported Ashkenazi Jewish ancestry; age at diagnosis; biopsy Gleason grade coded as a continuous variable $(1=$ Gleason $<=6$, $2=$ Gleason $7,3=$ Gleason $>=8)$; and clinical stage coded as a continuous variable $(1=\mathrm{T} 1,2=\mathrm{T} 2,3=\mathrm{T} 3 / 4)$.

Collection of blood samples for genetic testing began in 2000, and therefore, some cases diagnosed before 2000, and who died before 2000 (or who did not participate in blood sampling), were not included in this cohort. This scenario is referred to statistically as 'left truncation.' To account for this, we left-censored the interval from diagnosis to blood draw for each patient.

To address issues of multiple testing, by examining 61 SNPs and applying a Bonferroni correction, statistical significance was defined as $P<0.0008$. All statistical analyses were conducted using Plink (v1.07) and R (v2.9.1) as we have previously described (Willis et al, 2012).

\section{RESULTS}

One thousand three hundred and fifty-four patients were genotyped. Patient characteristics are presented in Table 3. The median age at diagnosis was 66 years (y) and median pre-operative PSA was $7.3 \mathrm{ng} \mathrm{ml}^{-1}$. Treatment at presentation was based on patient and physician preference. The majority of patients (93\%) were treated with curative intent: 466 (34\%) underwent radical prostatectomy with $804(59 \%)$ receiving radiotherapy (RT) with or without antiandrogen therapy. A majority of patients (61\%) had biopsy Gleason score $\geqslant 7$, and $53 \%$ of patients with available clinical staging information had $\geqslant \mathrm{T} 2$ disease. Median (interquartile range) follow-up for survivors was $10.4 \mathrm{y}$ (7.2-13.8). At last follow-up, BR was documented in 671 patients (49\%), CM in 313 (23\%), with 194 (14\%) individuals having died from PCa. Median (interquartile range) BR-free survival was 8.1y (2.6-not reached) and median time to CM 21.4y (11.7-23.3). At 5y after PCa diagnosis, $98 \%$ of the study population were alive, $91 \%$ at $10 \mathrm{y}, 76 \%$ at $15 \mathrm{y}$ and $62 \%$ at $20 \mathrm{y}$.

Univariate associations between susceptibility loci and PCa outcomes $(P<0.05)$ are summarised in Table 4 . In all, 2 of 61 SNPs, rs13385191 and rs339331, were associated with an increased risk of BR $(P<0.05)$. Three SNPs were associated with CM $(P<0.05)$; rs13385191 associated with an increased risk of CM (hazard ratio $(\mathrm{HR})=1.26)$, with rs9284813 and rs11067228 both associated with decreased risk of $\mathrm{CM}(\mathrm{HR}=0.75$ and 0.74 , respectively).

Seven SNPs showed associations on multivariable analysis with clinical end points $(P<0.05)$. Again rs13385191 $(\mathrm{HR}=1.36$; $95 \% \quad \mathrm{CI}=1.03-1.81 ; \quad P=0.02) \quad$ and $\quad \mathrm{rs} 339331 \quad(\mathrm{HR}=1.47$; $95 \% \mathrm{CI}=1.04-2.08 ; P=0.02)$ were associated with an increased risk of BR. Four SNPs, rs13385191, rs1894292, rs17178655 and rs11067228, were associated with $\mathrm{CM}$, with different directions of effect and $\mathrm{rs} 11902236(\mathrm{HR}=0.78 ; 95 \% \mathrm{CI}=0.62-0.98 ; P=0.03)$ and $\mathrm{rs} 4857841(\mathrm{HR}=0.78 ; 95 \% \mathrm{CI}=0.62-0.98 ; P=0.04)$ with PCSM (Table 5). Of note, none of these associations were significant after a Bonferroni correction for multiple testing was applied $(P<0.0008)$.

We also asked if any of the SNPs were associated with PSA at diagnosis. One SNP, rs17632542, was significant after multiple test correction $\left(P=1.7 \times 10^{-5}\right)$, with carriers of the risk allele [C] more likely to have lower PSA levels at diagnosis (Figure 1). 
Table 1. Prostate cancer risk polymorphisms genotyped and analysed in study cohort

\begin{tabular}{|c|c|c|c|c|c|c|c|}
\hline SNP & Chr & Pos & Major/minor allele & $\mathrm{MAF}^{\mathrm{a}}$ & Per-allele OR ${ }^{\mathrm{b}}$ & Candidate gene & Reference \\
\hline rs1218582 & $1 q 21$ & 153100807 & $\mathrm{AG}$ & 0.45 & $1.06(1.03-1.09)$ & KCNN3 & Eeles et al, 2013 \\
\hline rs4245739 & $1 q 32$ & 202785465 & $A C$ & 0.25 & $0.91(0.88-0.95)$ & MDM4-PIK3C2B & Eeles et al, 2013 \\
\hline rs10187424 & $2 p 11$ & 85647807 & AG & 0.41 & $0.92(0.89-0.94)$ & GGCX-VAMP8 & Kote-Jarai et al, 2011 \\
\hline rs1465618 & $2 p 21$ & 43465600 & GA & 0.23 & $1.08(1.03-1.12)$ & THADA & Eeles et al, 2013 \\
\hline rs6545977 & $2 p 15$ & 63154668 & GA & NR & NR & OTX1—RPL27P5 & Eeles et al, 2013 \\
\hline rs13385191 & $2 p 24$ & 20751746 & GA & 0.40 & $1.15(1.10-1.21)$ & C2orf43 & Takata et al, 2010 \\
\hline rs11902236 & $2 p 24$ & 10035319 & GA & 0.27 & $1.07(1.03-1.10)$ & TAF1B:GRHL1 & Eeles et al, 2013 \\
\hline rs3771570 & $2 q 37$ & 242031537 & GA & 0.15 & $1.12(1.08-1.17)$ & FARP2 & Eeles et al, 2013 \\
\hline rs7584330 & $2 q 37$ & 238051966 & TC & 0.22 & $1.06(1.02-1.09)$ & COL6A3 - MLPH & Kote-Jarai et al, 2011 \\
\hline rs7629490 & $3 p 11$ & 87324187 & CT & NR & $1.06(1.04-1.09)$ & VGLL3 - CHMP2B & Schumacher et al, 2011 \\
\hline rs9284813 & $3 p 12$ & 87234859 & AG & NR & $\mathrm{NR}$ & VGLL3 - CHMP2B & Takata et al, 2010 \\
\hline rs7611694 & $3 q^{13}$ & 114758314 & $A C$ & 0.41 & $0.91(0.88-0.93)$ & SIDT1 & Eeles et al, 2013 \\
\hline rs6763931 & $3 q 23$ & 142585522 & CT & 0.45 & $1.04(1.01-1.07)$ & ZBTB38 & Kote-Jarai et al, 2011 \\
\hline rs4857841 & $3 q 21$ & 129529333 & GA & 0.30 & $1.13(1.08-1.18)$ & EEFSEC & Lindstrom et al, 2012 \\
\hline rs1894292 & $4 q^{13}$ & 74714193 & GA & 0.48 & $0.91(0.89-0.94)$ & AFM, RASSF6 & Eeles et al, 2013 \\
\hline rs17021918 & $4 q 22$ & 95781900 & $\mathrm{CT}$ & 0.34 & $0.90(0.87-0.93)$ & PDLIM5 & Eeles et al, 2013 \\
\hline rs12500426 & $4 q 22$ & 95733632 & CA & 0.46 & $1.08(1.05-1.12)$ & PDLIM5 & Eeles et al, 2013 \\
\hline rs7679673 & $4 q 24$ & 106280983 & $\mathrm{CA}$ & 0.45 & $0.91(0.88-0.94)$ & RPL6P14-TET2 & Eeles et al, 2013 \\
\hline rs2121875 & $5 p 12$ & 44401301 & TG & 0.34 & $1.05(1.02-1.08)$ & FGF10 & Kote-Jarai et al, 2011 \\
\hline rs4466137 & $5 q^{14}$ & 83021495 & TG & NR & NR & HAPLN1 & Murabito et al, 2007 \\
\hline rs2242652 & $5 p 15$ & 1333027 & GA & 0.19 & $0.87(0.84-0.90)$ & TERT & Kote-Jarai et al, 2011 \\
\hline rs12653946 & $5 p 15$ & 1948829 & CT & 0.50 & $1.31(1.20-1.42)$ & $I R X 4-I R X 2$ & Takata et al, 2010 \\
\hline rs6869841 & $5 q 35$ & 172872032 & GA & 0.21 & $1.07(1.04-1.11)$ & FAM44B (BOD1) & Eeles et al, 2013 \\
\hline rs2273669 & $6 p 21$ & 109391882 & AG & 0.15 & $1.07(1.03-1.11)$ & ARMC2,SESN1 & Eeles et al, 2013 \\
\hline rs339331 & $6 q 22$ & 117316745 & $\mathrm{TC}$ & 0.31 & $1.28(1.17-1.40)$ & GPRC6A;RFX6 & Takata et al, 2010 \\
\hline rs1933488 & $6 q 25$ & 153482772 & AG & 0.41 & $0.89(0.87-0.92)$ & RSG17 & Eeles et al, 2013 \\
\hline rs651164 & $6 q 25$ & 160551785 & GA & NR & $0.87(0.83-0.91)$ & LOC100289162 & Schumacher et al, 2011 \\
\hline rs12155172 & $7 p 15$ & 20767731 & GA & 0.20 & $1.05(0.98-1.10)$ & RPS26P30-ASS1P11-SP8 & Eeles et al, 2013 \\
\hline rs2928679 & $8 p 21$ & 23494920 & CT & 0.42 & $1.05(1.01-1.09)$ & SLC25A37 NKX3-1 & Eeles et al, 2013 \\
\hline rs1512268 & $8 p 21$ & 23582408 & GA & 0.45 & $1.18(1.14-1.22)$ & SLC25A37 - NKX3-1 & Eeles et al, 2013 \\
\hline rs11135910 & $8 p 21$ & 25948059 & GA & 0.16 & $1.11(1.07-1.16)$ & EBF2 & Eeles et al, 2013 \\
\hline rs10086908 & $8 q 24$ & 128011937 & TC & 0.3 & $0.87(0.81-0.94)$ & POU5F1B, MYC & Eeles et al, 2013 \\
\hline rs 12543663 & $8 q 24$ & 127993841 & $A C$ & 0.33 & $1.08(1.00-1.16)$ & LOC727677, MYC & Al Olama AA et al, 2009 \\
\hline rs13252298 & $8 q 24$ & 128164338 & $A G$ & NR & $0.89(0.85-0.95)$ & FAM84B - SRRM1P1 & Schumacher et al, 2011 \\
\hline rs445114 & $8 q 24$ & 128392363 & $\mathrm{TC}$ & 0.36 & $1.14(1.10-1.19)$ & SRRM1P1 - POU5F1B & Gudmundsson et al, 2010 \\
\hline rs16902094 & $8 q 24$ & 128320346 & AG & 0.15 & $1.21(1.15-1.26)$ & SRRM1P1 - POU5F1B & Gudmundsson et al, 2010 \\
\hline rs817826 & $9 q 31$ & 107235855 & TC & 0.10 & $1.43(1.17-1.77)$ & RAD23B-KLF4 & Xu et al, 2010 \\
\hline rs2252004 & $10 q 26$ & 122844709 & GT & 0.23 & $1.16(1.10-1.22)$ & $N R$ & Akamatsu et al, 2012 \\
\hline rs11199874 & $10 q 26$ & 123022509 & GA & 0.29 & $2.9(2.1-4.1)$ & RPL19P16-FGFR2 & Nam et al, 2006 \\
\hline rs1938781 & $11 q 12$ & 58915110 & TC & 0.3 & $1.16(1.11-1.21)$ & FAM111A & Akamatsu et al, 2012 \\
\hline rs11228565 & $11 q 13$ & 68735156 & GA & 0.2 & $1.23(1.16-1.31)$ & TPCN2 - MYEOV & Gudmundsson et al, 2010 \\
\hline rs7127900 & $11 p 15$ & 2233574 & GA & 0.20 & $1.22(1.17-1.27)$ & IGF2-INS & Eeles et al, 2013 \\
\hline rs11568818 & $11 q 22$ & 101906871 & AG & 0.44 & $0.91(0.88-0.94)$ & MMP7 & Eeles et al, 2013 \\
\hline rs10875943 & $12 q 13$ & 47962277 & TC & 0.31 & $1.07(1.04-1.10)$ & TUBA1C-PRPH & Kote-Jarai et al, 2011 \\
\hline rs1270884 & $12 q 24$ & 113169954 & GA & 0.49 & $1.07(1.04-1.10)$ & TBX5 & Eeles et al, 2013 \\
\hline rs1529276 & $13 q 33$ & 102726008 & TA & NR & NR & SLC10A2-RPL7P45 & Murabito et al, 2007 \\
\hline rs8008270 & $14 q 22$ & 52442080 & GA & 0.18 & $0.89(0.86-0.93)$ & FERMT2 & Eeles et al, 2013 \\
\hline rs7141529 & $14 q 24$ & 68196497 & AG & 0.50 & $1.09(1.06-1.12)$ & RAD51B & Eeles et al, 2013 \\
\hline rs11650494 & $17 q 12$ & 44700185 & GA & 0.08 & $1.15(1.09-1.22)$ & $\begin{array}{c}\text { GNGT2, ABI3, PHB, } \\
\text { SPOP, HOXB13 }\end{array}$ & Eeles et al, 2013 \\
\hline rs7241993 & $18 q 23$ & 74874961 & GA & 0.30 & $0.92(0.89-0.95)$ & SALL3 & Eeles et al, 2013 \\
\hline rs8102476 & $19 q 13$ & 38735613 & CT & 0.46 & $1.12(1.08-1.15)$ & DPF1 - PPP1R14A & Gudmundsson et al, 2010 \\
\hline
\end{tabular}


Table 1. (Continued)

\begin{tabular}{|c|c|c|c|c|c|c|c|}
\hline SNP & Chr & Pos & Major/minor allele & MAF $^{a}$ & Per-allele OR ${ }^{b}$ & Candidate gene & Reference \\
\hline rs103294 & $19 q 13$ & 54797848 & $\mathrm{TC}$ & 0.30 & $1.28(1.21-1.45)$ & LILRA3 & Xu et al, 2010 \\
\hline rs2427345 & $20 q 13$ & 60449006 & GA & 0.37 & $0.94(0.91-0.97)$ & GATAS, CABLES2 & Eeles et al, 2013 \\
\hline rs6062509 & $20 q 13$ & 61833007 & $A C$ & 0.30 & $0.89(0.66-0.92)$ & ZGPAT & Eeles et al, 2013 \\
\hline rs742134 & $22 q 13$ & 41842773 & $A G$ & NR & $1.16(1.01-1.23)$ & $B I K$ & Schumacher et al, 2011 \\
\hline rs5759167 & $22 q 13$ & 41830156 & GT & 0.47 & $0.86(0.83-0.88)$ & RPS25P10-BIK & Eeles et al, 2013 \\
\hline \multicolumn{8}{|c|}{ 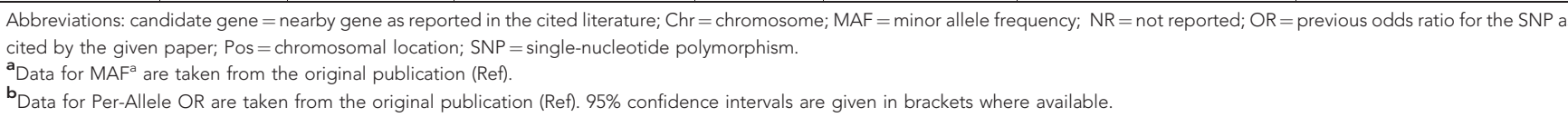 } \\
\hline
\end{tabular}

Table 2. PSA-associated polymorphisms genotyped and analysed in study cohort

\begin{tabular}{|c|c|c|c|c|c|c|c|}
\hline SNP & Chr & Pos & Risk allele & RAF & Increase per allele (\%) & Candidate gene & Reference \\
\hline rs401681 & $5 p 15$ & 1375087 & $\mathrm{C}$ & 0.55 & 7 & CLPTM1L & Gudmundsson et al, 2010 \\
\hline rs10788160 & $10 q 26$ & 123023539 & A & 0.31 & 10.2 & RPL19P16 - FGFR2 & Gudmundsson et al, 2010 \\
\hline rs17632542 & $19 q 13$ & 51361757 & $\mathrm{~T}$ & 0.91 & 39.1 & KLK3 & Gudmundsson et al, 2010 \\
\hline rs11067228 & $12 q 24$ & 113556980 & A & 0.56 & 8.3 & OSTF1P1-TBX3 & Gudmundsson et al, 2010 \\
\hline rs17178655 & $10 q^{11}$ & 51231805 & A & 0.23 & NR & MSMB & Xu et al, 2010 \\
\hline
\end{tabular}

\begin{tabular}{|c|c|}
\hline Characteristic & Median (IQR) or frequency (\%) \\
\hline Age at diagnosis (years) & $66(60-71)$ \\
\hline \multicolumn{2}{|l|}{ Year of diagnosis } \\
\hline 1988-1995 & $393(29 \%)$ \\
\hline 1996-2000 & $558(41 \%)$ \\
\hline 2001-2006 & 403 (30\%) \\
\hline Pre-treatment PSA $\left(\mathrm{ng} \mathrm{ml}^{-1}\right)$ & $7.3(4.2-12.9)$ \\
\hline Family history $\mathrm{PCa}$ & $141(11 \%)$ \\
\hline \multicolumn{2}{|l|}{ Biopsy gleason grade } \\
\hline$\leqslant 6$ & $503(37 \%)$ \\
\hline 7 & $532(39 \%)$ \\
\hline$\geqslant 8$ & $288(22 \%)$ \\
\hline Unknown & $31(2 \%)$ \\
\hline \multicolumn{2}{|l|}{ Clinical stage } \\
\hline T1 & $576(42 \%)$ \\
\hline T2 & $512(38 \%)$ \\
\hline T3/4 & $201(15 \%)$ \\
\hline Unknown & $65(5 \%)$ \\
\hline \multicolumn{2}{|l|}{ Type of treatment } \\
\hline Radical prostatectomy & 466 (34\%) \\
\hline $\begin{array}{l}\text { Radiotherapy } \pm \text { androgen } \\
\text { deprivation }\end{array}$ & $804(59 \%)$ \\
\hline Androgen deprivation alone/WW & $84(7 \%)$ \\
\hline \multicolumn{2}{|c|}{$\begin{array}{l}\text { Abbreviations: } 1 \mathrm{QR}=\text { interquartile range; } \mathrm{PCa}=\text { prostate cancer; } \mathrm{PSA}=\text { prostate-specifi } \\
\text { antigen; } W W=\text { watchful waiting. }\end{array}$} \\
\hline
\end{tabular}

\section{DISCUSSION}

Several existing PCa nomograms incorporating clinico-pathological parameters such as Gleason score, TNM stage and PSA aid in predicting likelihood of disease recurrence (Kattan et al, 1998; Stephenson et al, 2009), however, they are limited in their prognostic capabilities. Novel biomarkers to identify aggressiveness of disease and likelihood of recurrence are required. Although much focus is currently being placed on analysis of somatic mutations in contributing to these predictive models (Erho et al, 2013;
Table 4. Univariate associations between SNPs and PCa outcomes under a codominant model $(P<0.05$ by the 2 df test)

\begin{tabular}{|c|c|c|c|c|c|c|}
\hline SNP & $\mathrm{Chr}$ & Gene & $\begin{array}{l}\text { Minor } \\
\text { allele }\end{array}$ & MAF & $\begin{array}{c}\text { HR } \\
(95 \% \mathrm{Cl})\end{array}$ & $\begin{array}{c}P \\
\text { value }\end{array}$ \\
\hline \multicolumn{7}{|c|}{ Biochemical recurrence } \\
\hline rs13385191 & 2 & C2orf43 & A & 0.27 & $\begin{array}{c}1.36 \\
(1.02-1.81)\end{array}$ & 0.03 \\
\hline rs339331 & 6 & $\begin{array}{c}R F X 6 / \\
\text { GPRC6A }\end{array}$ & C & 0.15 & $\begin{array}{c}1.45 \\
(1.03-2.02)\end{array}$ & 0.02 \\
\hline \multicolumn{7}{|c|}{ Castrate metastasis } \\
\hline rs13385191 & 2 & C2orf43 & A & 0.27 & $\begin{array}{c}1.28 \\
(1.02-1.60)\end{array}$ & 0.02 \\
\hline rs9284813 & 3 & VGLL3 & G & 0.26 & $\begin{array}{c}0.75 \\
(0.57-0.98)\end{array}$ & 0.03 \\
\hline rs11067228 & 12 & OSTF1P1 & G & 0.48 & $\begin{array}{c}0.74 \\
(0.60-0.93)\end{array}$ & 0.009 \\
\hline
\end{tabular}

Karnes et al, 2013), germline genetic variants have certain unique advantages. Knowing the inherited genetic predisposition of an individual to develop recurrent disease and metastatic progression at the time of diagnosis would clearly inform decision making regarding best initial treatment strategy and the intensity and approach to follow-up.

Since the first PCa GWAS in 2006 (Amundadottir et al, 2006) up through the most recent addition of a further 23 susceptibility loci by the PRACTICAL consortium in 2013 (Eeles et al, 2013), over 75 SNPs known to be associated with PCa risk have been identified. The ability of these susceptibility loci, however, to predict disease aggressiveness and clinical outcomes is less clear. Although several studies have reported associations with diseasespecific outcomes, results are often conflicting and inconsistent (Penney et al, 2009; Wiklund et al, 2009; Gallagher et al, 2010; Pomerantz et al, 2011; Szulkin et al, 2012). Most recently, Shui et al analysed the association of 47 PCa susceptibility loci with PCSM in a large cohort with over 1000 events and reported association of 
Table 5. Multivariate associations between SNPs and PCa

outcomes

\begin{tabular}{|c|c|c|c|c|c|c|}
\hline SNP & Chr & Gene & $\begin{array}{l}\text { Minor } \\
\text { Allele }\end{array}$ & MAF & $\begin{array}{c}\text { HR } \\
(95 \% \mathrm{Cl}) \\
\end{array}$ & $\begin{array}{c}P \\
\text { value }\end{array}$ \\
\hline \multicolumn{7}{|c|}{ Biochemical recurrence } \\
\hline rs13385191 & 2 & C2orf43 & A & 0.27 & $1.36(1.03-1.81)$ & 0.02 \\
\hline rs339331 & 6 & $\begin{array}{c}\text { RFX6/ } \\
\text { GPRC6A }\end{array}$ & C & 0.15 & $1.47(1.04-2.08)$ & 0.02 \\
\hline \multicolumn{7}{|c|}{ Castrate metastasis } \\
\hline rs13385191 & 2 & C2orf43 & A & 0.27 & $1.28(1.03-1.60)$ & 0.02 \\
\hline rs1894292 & 4 & AFM,RASSF6 & A & 0.42 & $1.25(1.01-1.54)$ & 0.03 \\
\hline rs17178655 & 10 & MSMB & A & 0.21 & $0.73(0.55-0.97)$ & 0.03 \\
\hline rs11067228 & 12 & OSTF1P1 & G & 0.48 & $0.79(0.63-0.99)$ & 0.04 \\
\hline \multicolumn{7}{|c|}{ Prostate cancer-specific mortality } \\
\hline rs11902236 & 2 & TAF1B:GRHL1 & A & 0.34 & $0.78(0.62-0.98)$ & 0.03 \\
\hline rs4857841 & 3 & EEFSEC & A & 0.31 & $0.78(0.62-0.98)$ & 0.04 \\
\hline \multicolumn{7}{|c|}{$\begin{array}{l}\text { Abbreviations: } \mathrm{Chr}=\text { chromosome; } \mathrm{Cl}=\text { confidence interval; } \mathrm{HR}=\text { hazard ratios; } \\
\mathrm{MAF}=\text { minor allele frequency; } \mathrm{SNP}=\text { single-nucleotide polymorphism. NOTE: Each SNP } \\
\text { was individually assessed in separate multivariable models, controlling for age at prostate } \\
\text { cancer diagnosis, } \mathrm{PSA} \text { at diagnosis, clinical stage and biopsy Gleason grade. }\end{array}$} \\
\hline
\end{tabular}

eight SNPs with disease-specific death (Shui et al, 2014). In this same study, however, susceptibility loci were not able to distinguish aggressive $v s$ non-aggressive disease (Shui et al, 2014).

We believe our current study is the first to assess a large number of susceptibility loci with respect to all three clinical end points with extensive follow-up (median 10.4y). We found evidence of associations of several SNPs with all three clinical end points on both univariate and multivariable analyses $(P<0.05)$. Importantly, however, when incorporating a Bonferonni correction for multiple testing $(P<0.0008)$, the only persistent significant association was with rs17632542, a previously reported KLK3 variant (Gudmundsson et al, 2010; Klein et al, 2010; Kote-Jarai et al, 2011; Parikh et al, 2011), and PSA levels at diagnosis. Thus, the evidence for association at the other SNPs is only suggestive at this point and will need to be replicated in other studies.

rs17632542 lies within exon 4 of the KLK3 gene and has also previously been associated with PCa risk (Kote-Jarai et al, 2011; Parikh et al, 2011; Penney et al, 2011; Klein et al, 2012; Knipe et al, 2014). The minor allele (C) causes a non-synonymous aminoacid change from isoleucine to threonine at position 179 (Ile179Thr). In our analysis, carriers of the C allele had a lower PSA at diagnosis; however, there were no associations seen with age at diagnosis, disease stage, Gleason grade, family history of PCa or any of the disease-specific clinical end points. This direction of effect is consistent with other studies such as by Gudmundsson et al who reported carriers of the rs17632542-T allele as having higher PSA levels (Gudmundsson et al, 2010). Interestingly, we have previously reported that $\mathrm{rs} 17632542-\mathrm{C}$ is associated with decreased PCa risk, $(\mathrm{OR}=0.64(\mathrm{CI}=0.51-0.81) P=0.00019)$. It is plausible that harbouring the rare allele of rs17632542 (C) leads to a direct effect on the function of the PSA protein, possibly through regulatory effects (on transcription of the gene), through altered protein stability or effect on antigenicity and as such detectability in PSA tests. It is also plausible that patients with the rare allele may be less likely to undergo biopsy subsequent to PSA screening because of lower PSA levels, although they may harbour asymptomatic and indolent PCa.

Two other PSA-related SNPs showed associations on multivariable analysis $(P<0.05)$. rs11067228 is located in a linkage disequlibrium block that contains the genes TBX3 (T-Box Transcription Factor 3) and OSTF1P1 (Osteoclast Stimulating Factor 1 Pseudogene 1), with the common allele (A) being previously associated with higher PSA levels (Gudmundsson et al, 2010). The same study reported no association, however, with PCa but an association with a greater probability of having a normal

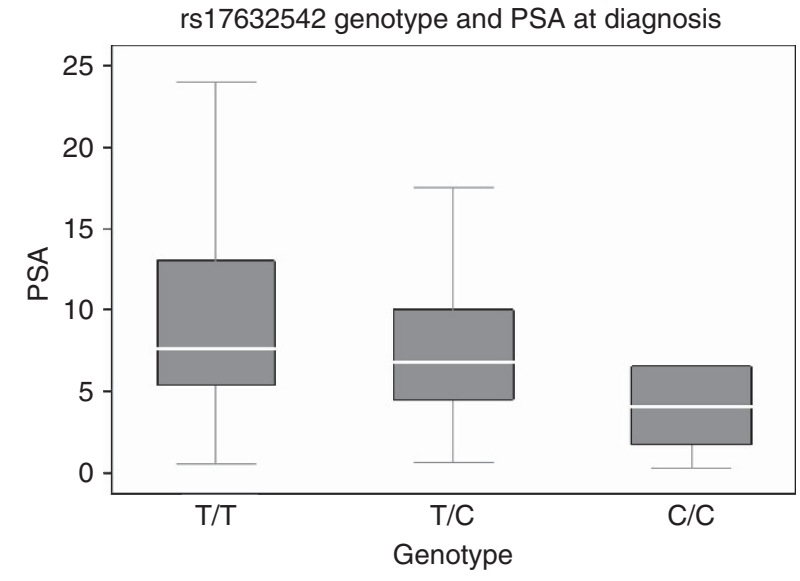

Excludes outside values

Figure 1. Box plot graph for rs17632542 (KLK3) illustrating PSA level at diagnosis with respect to allele (Common T, Het TC, Rare C).

prostate biopsy (Gudmundsson et al, 2010). In our study, we observed rs11067228-G to be associated with a lesser chance of development of castrate-resistant disease ( $\mathrm{HR}=0.79$ $(\mathrm{CI}=0.63-0.99), P=0.04)$. rs17178655, an intronic variant in the microseminoprotein $\beta$ gene $(\beta-M S P)$, was also seen to be associated with development of $\mathrm{CM}$, with carriers of the minor allele (A) less likely to develop $\mathrm{CM}(\mathrm{HR}=0.73(\mathrm{CI}=0.55-0.97)$ $P=0.03$ ). This SNP had previously been reported by our group to be associated with semen levels of both free and total PSA $(P=0.0027)$ but interestingly not levels of $\beta$-MSP (Xu et al, 2010). In contrast with PSA, whereby risk of $\mathrm{PCa}$ increases with higher PSA levels, $\beta$-MSP levels measured in serum, urine and prostate tissue have been shown to be statistically significantly lower in men with $\mathrm{PCa}$ and even lower in men with aggressive disease (Nam et al, 2006; Whitaker et al, 2010).

rs13385191 is located in intron 6 of C2orf43 (chromosome 2 open reading frame 43$)$ and achieved significance $(P<0.05)$ on multivariable analysis for both clinical end points of $\mathrm{BR}(\mathrm{HR}=1.36$ $(\mathrm{CI}=1.03-1.81) P=0.02)$ and $\mathrm{CM}(\mathrm{HR}=1.28(\mathrm{CI}=1.03-1.60)$ $P=0.02)$. This SNP was initially reported by Takata et al in 2010 with the rare allele associated with increased risk of PCa in an Asian population $(\mathrm{OR}=1.15(\mathrm{CI}=1.10-1.21)$; Takata et al, 2010) and subsequently replicated in both European $(\mathrm{OR}=1.07$ $(\mathrm{CI}=1.02-1.12)$; Lindstrom et al, 2012) and Chinese populations $(\mathrm{OR}=1.33$ (CI = 1.11-1.58); Long et al, 2012). Recently, Shui et al (2014) reported association of rs13385191 with PCSM, however, with the opposite direction of effect $(\mathrm{OR}=0.88(\mathrm{CI}=0.78-1.00)$ $P=0.05$ ). C2orf 43 is a highly conserved gene (Long et al, 2012) and as such, may harbour important functional variants in or within close proximity to its location around $2 \mathrm{p} 24$.

There were four additional SNPs (rs339331, rs1894292, rs11902236, rs4857841), which showed associations with end points at $P<0.05$. Importantly, however, the allele conferring an increased risk of PCa from previous GWAS studies was, in our analysis, a predictor of less aggressive disease as measured by time to recurrence and disease-specific death (Table 4).

The above results and those from other similar analyses lead us to conclude that susceptibility loci that are associated with initiation and development of PCa are likely to differ from loci that predict disease progression and aggressiveness. The mechanisms and pathways contributing to a more aggressive disease phenotype are still elusive, and additional large-scale discovery studies focusing on disease-specific end points are required. Investigating the cumulative effect of PCa SNPs may well reveal more about the molecular mechanisms of PCa oncogenesis (Jiang et al, 2013). As we discover further risk loci, pathway analysis 
and computational statistical programmes will hopefully shed further light on these molecular mechanisms. In addition, we must also be aware that SNP function may vary among ethnic populations as has been suggested in other recent work (Jiang et al, 2014).

Our study has several limitations: although we report associations of a large selection of PCa risk loci, there are a number of reported GWAS SNPs that due to genotyping failures, were not included in the analysis. We also did not set out to discover any novel susceptibility loci or pathways. However as strengths, we utilised a large sample size with extended follow-up and granular phenotypic data, which includes detailed pathological and treatment variables.

\section{CONCLUSIONS}

The ability to discriminate individuals who are more or less likely to harbour an aggressive PCa phenotype and who are predisposed to disease recurrence has long been the focus of attention by the urologic oncology community. Existing nomograms are clinically useful but there is significant potential to increase their accuracy with addition of new biomarkers and individual genetic predictors. In this study, we confirmed that rs 17632542 in KLK3 is associated with PSA at diagnosis confirming reproducibility across multiple cohorts. No significant association was seen between loci and disease-specific end points when accounting for multiple testing. This provides further evidence that known PCa risk SNPs do not predict likelihood of disease progression. Further larger discovery analysis in cohorts with robust clinical end points are required to shed further light on germline predictors of disease recurrence to improve initial management and surveillance strategies.

\section{ACKNOWLEDGEMENTS}

This work has been funded by R01 CA175491 from the National Cancer Institute (RJK) and the Robert and Kate Niehaus Clinical Cancer Genetics Initiative at MSKCC (KO). We are grateful to all other ancillary and support staff from the Department of Clinical Genetics MSKCC for such support during the project.

\section{CONFLICT OF INTEREST}

The authors declare no conflict of interest.

\section{REFERENCES}

Akamatsu S, Takata R, Haiman CA, Takahashi A, Inoue T, Kubo M, Furihata M, Kamatani N, Inazawa J, Chen GK, Le Marchand L, Kolonel LN, Katoh T, Yamano Y, Yamakado M, Takahashi H, Yamada H, Egawa S, Fujioka T, Henderson BE, Habuchi T, Ogawa O, Nakamura Y, Nakagawa $H$ (2012) Common variants at 11q12, 10q26 and 3p11.2 are associated with prostate cancer susceptibility in Japanese. Nat Genet 44(S1): 426-429.

Al Olama AA, Kote-Jarai Z, Giles GG, Guy M, Morrison J, Severi G, Leongamornlert DA, Tymrakiewicz M, Jhavar S, Saunders E, Hopper JL, Southey MC, Muir KR, English DR, Dearnaley DP, Ardern-Jones AT, Hall AL, O'Brien LT, Wilkinson RA, Sawyer E, Lophatananon A. The UK Genetic Prostate Cancer Study Collaborators/British Association of Urological Surgeons' Section of Oncology, The UK Prostate testing for cancer and Treatment study (ProtecT Study) Collaborators, Horwich A, Huddart RA, Khoo VS, Parker CC, Woodhouse CJ, Thompson A, Christmas T, Ogden C, Cooper C, Donovan JL, Hamdy FC, Neal DE, Eeles RA, Easton DF (2009) Multiple loci on 8q24 associated with prostate cancer susceptibility. Nat Genet 41: 1058-1060.

Amundadottir LT, Sulem P, Gudmundsson J, Helgason A, Baker A, Agnarsson BA, Sigurdsson A, Benediktsdottir KR, Cazier JB, Sainz J,
Jakobsdottir M, Kostic J, Magnusdottir DN, Ghosh S, Agnarsson K, Birgisdottir B, Le roux L, Olafsdottir A, Blondal T, Andresdottir M, Gretarsdottir OS, Bergthorsson JT, Gudbjartsson D, Gylfason A, Thorleifsson G, Manolescu A, Kristjansson K, Geirsson G, Isaksson H, Douglas J, Johansson JE, Balter K, Wiklund F, Montie JE, Yu X, Suarez BK, Ober C, Cooney KA, Gronberg H, Catalona WJ, Einarsson GV, Barkardottir RB, Gulcher JR, Kong A, Thorsteinsdottir U, Stefansson K (2006) A common variant associated with prostate cancer in European and African populations. Nat Genet 38: 652-658.

Eeles RA, Olama AA, Benlloch S, Saunders EJ, Leongamornlert DA, Tymrakiewicz M, Ghoussaini M, Luccarini C, Dennis J, Jugurnauth-Little S, Dadaev T, Neal DE, Hamdy FC, Donovan JL, Muir K, Giles GG, Severi G, Wiklund F, Gronberg H, Haiman CA, Schumacher F, Henderson BE, Le Marchand L, Lindstrom S, Kraft P, Hunter DJ, Gapstur S, Chanock SJ, Berndt SI, Albanes D, Andriole G, Schleutker J, Weischer M, Canzian F, Riboli E, Key TJ, Travis RC, Campa D, Ingles SA, John EM, Hayes RB, Pharoah PD, Pashayan N, Khaw KT, Stanford JL, Ostrander EA, Signorello LB, Thibodeau SN, Schaid D, Maier C, Vogel W, Kibel AS, Cybulski C, Lubinski J, Cannon-Albright L, Brenner H, Park JY, Kaneva R, Batra J, Spurdle AB, Clements JA, Teixeira MR, Dicks E, Lee A, Dunning AM, Baynes C, Conroy D, Maranian MJ, Ahmed S, Govindasami K, Guy M, Wilkinson RA, Sawyer EJ, Morgan A, Dearnaley DP, Horwich A, Huddart RA, Khoo VS, Parker CC, Van As NJ, Woodhouse CJ, Thompson A, Dudderidge T, Ogden C, Cooper CS, Lophatananon A, Cox A, Southey MC, Hopper JL, English DR, Aly M, Adolfsson J, Xu J, Zheng SL, Yeager M, Kaaks R, Diver WR, Gaudet MM, Stern MC, Corral R, Joshi AD, Shahabi A, Wahlfors T, Tammela TL, Auvinen A, Virtamo J, Klarskov P, Nordestgaard BG, Røder MA, Nielsen SF, Bojesen SE, Siddiq A, Fitzgerald LM, Kolb S, Kwon EM, Karyadi DM, Blot WJ, Zheng W, Cai Q, McDonnell SK, Rinckleb AE, Drake B, Colditz G, Wokolorczyk D, Stephenson RA, Teerlink C, Muller H, Rothenbacher D, Sellers TA, Lin HY, Slavov C, Mitev V, Lose F, Srinivasan S, Maia S, Paulo P, Lange E, Cooney KA, Antoniou AC, Vincent D, Bacot F, Tessier DC. COGS-Cancer Research UK GWAS-ELLIPSE (part of GAME-ON) InitiativeAustralian Prostate Cancer BioresourceUK Genetic Prostate Cancer Study Collaborators/ British Association of Urological Surgeons' Section of OncologyUK ProtecT (Prostate testing for cancer and Treatment) Study CollaboratorsPRACTICAL (Prostate Cancer Association Group to Investigate Cancer-Associated Alterations in the Genome) ConsortiumKote-Jarai Z, Easton DF (2013) Identification of 23 new prostate cancer susceptibility loci using the iCOGS custom genotyping array. Nat Genet 45: 385-391391e1-2.

Erho N, Crisan A, Vergara IA, Mitra AP, Ghadessi M, Buerki C, Bergstralh EJ, Kollmeyer T, Fink S, Haddad Z, Zimmermann B, Sierocinski T, Ballman KV, Triche TJ, Black PC, Karnes RJ, Klee G, Davicioni E, Jenkins RB (2013) Discovery and validation of a prostate cancer genomic classifier that predicts early metastasis following radical prostatectomy. PLoS One 8: e66855.

Gabriel S, Ziaugra L, Tabbaa D (2009) SNP genotyping using the Sequenom MassARRAY iPLEX platform. Curr Protoc Hum Genet Chapter 2: Unit 212.

Gallagher DJ, Vijai J, Cronin AM, Bhatia J, Vickers AJ, Gaudet MM, Fine S, Reuter V, Scher HI, Hallden C, Dutra-Clarke A, Klein RJ, Scardino PT, Eastham JA, Lilja H, Kirchhoff T, Offit K (2010) Susceptibility loci associated with prostate cancer progression and mortality. Clin Cancer Res 16: $2819-2832$.

Gudmundsson J, Besenbacher S, Sulem P, Gudbjartsson DF, Olafsson I, Arinbjarnarson S, Agnarsson BA, Benediktsdottir KR, Isaksson HJ, Kostic JP, Gudjonsson SA, Stacey SN, Gylfason A, Sigurdsson A, Holm H, Bjornsdottir US, Eyjolfsson GI, Navarrete S, Fuertes F, Garcia-Prats MD, Polo E, Checherita IA, Jinga M, Badea P, Aben KK, Schalken JA, Van Oort IM, Sweep FC, Helfand BT, Davis M, Donovan JL, Hamdy FC, Kristjansson K, Gulcher JR, Masson G, Kong A, Catalona WJ, Mayordomo JI, Geirsson G, Einarsson GV, Barkardottir RB, Jonsson E, Jinga V, Mates D, Kiemeney LA, Neal DE, Thorsteinsdottir U, Rafnar T, Stefansson K (2010) Genetic correction of PSA values using sequence variants associated with PSA levels. Sci Transl Med 2: 62ra92.

Jiang J, Cui W, Vongsangnak W, Hu G, Shen B (2013) Post genome-wide association studies functional characterization of prostate cancer risk loci. BMC Genomics 14(Suppl 8): S9.

Jiang J, Jia P, Shen B, Zhao Z (2014) Top associated SNPs in prostate cancer are significantly enriched in cis-expression quantitative trait loci and at transcription factor binding sites. Oncotarget 5: 6168-6177. 
Karnes RJ, Bergstralh EJ, Davicioni E, Ghadessi M, Buerki C, Mitra AP, Crisan A, Erho N, Vergara IA, Lam LL, Carlson R, Thompson DJ, Haddad Z, Zimmermann B, Sierocinski T, Triche TJ, Kollmeyer T, Ballman KV, Black PC, Klee GG, Jenkins RB (2013) Validation of a genomic classifier that predicts metastasis following radical prostatectomy in an at risk patient population. J Urol 190: 2047-2053.

Kattan MW, Eastham JA, Stapleton AM, Wheeler TM, Scardino PT (1998) A preoperative nomogram for disease recurrence following radical prostatectomy for prostate cancer. J Natl Cancer Inst 90: 766-771.

Klein RJ, Hallden C, Cronin AM, Ploner A, Wiklund F, Bjartell AS, Stattin P, Xu J, Scardino PT, Offit K, Vickers AJ, Gronberg H, Lilja H (2010) Blood biomarker levels to aid discovery of cancer-related single-nucleotide polymorphisms: kallikreins and prostate cancer. Cancer Prev Res (Phila) 3: 611-619.

Klein RJ, Hallden C, Gupta A, Savage CJ, Dahlin A, Bjartell A, Manjer J, Scardino PT, Ulmert D, Wallstrom P, Vickers AJ, Lilja H (2012) Evaluation of multiple risk-associated single nucleotide polymorphisms versus prostate-specific antigen at baseline to predict prostate cancer in unscreened men. Eur Urol 61: 471-477.

Knipe DW, Evans DM, Kemp JP, Eeles R, Easton DF, Kote-Jarai Z, Al Olama AA, Benlloch S, Donovan JL, Hamdy FC, Neal DE, Davey Smith G, Lathrop M, Martin RM (2014) Genetic variation in protein specific antigen detected prostate cancer and the effect of control selection on genetic association studies. Cancer Epidemiol Biomarkers Prev 23(7): 1356-1365.

Kote-Jarai Z, Amin Al Olama A, Leongamornlert D, Tymrakiewicz M, Saunders E, Guy M, Giles GG, Severi G, Southey M, Hopper JL, Sit KC, Harris JM, Batra J, Spurdle AB, Clements JA, Hamdy F, Neal D, Donovan J, Muir K, Pharoah PD, Chanock SJ, Brown N, Benlloch S, Castro E, Mahmud N, O'brien L, Hall A, Sawyer E, Wilkinson R, Easton DF, Eeles RA (2011) Identification of a novel prostate cancer susceptibility variant in the KLK3 gene transcript. Hum Genet 129: 687-694.

Lindstrom S, Schumacher FR, Campa D, Albanes D, Andriole G, Berndt SI, Bueno-De-Mesquita HB, Chanock SJ, Diver WR, Ganziano JM, Gapstur SM, Giovannucci E, Haiman CA, Henderson B, Hunter DJ, Johansson M, Kolonel LN, Le Marchand L, Ma J, Stampfer M, Stevens VL, Trichopoulos D, Virtamo J, Willett WC, Yeager M, Hsing AW, Kraft P (2012) Replication of five prostate cancer loci identified in an Asian population-results from the NCI Breast and Prostate Cancer Cohort Consortium (BPC3). Cancer Epidemiol Biomarkers Prev 21: 212-216.

Long QZ, Du YF, Ding XY, Li X, Song WB, Yang Y, Zhang P, Zhou JP, Liu XG (2012) Replication and fine mapping for association of the C2orf43, FOXP4, GPRC6A and RFX6 genes with prostate cancer in the Chinese population. PLoS One 7: e37866.

Murabito JM, Rosenberg CL, Finger D, Kreger BE, Levy D, Splansky GL, Antman K, Hwang SJ (2007) A genome-wide association study of breast and prostate cancer in the NHLBI's Framingham Heart Study. BMC Med Genet 8(Suppl 1): S6.

Nam RK, Reeves JR, Toi A, Dulude H, Trachtenberg J, Emami M, Daigneault L, Panchal C, Sugar L, Jewett MA, Narod SA (2006) A novel serum marker, total prostate secretory protein of 94 amino acids, improves prostate cancer detection and helps identify high grade cancers at diagnosis. J Urol 175: 1291-1297.

Nielsen ME, Makarov DV, Humphreys E, Mangold L, Partin AW, Walsh PC (2008) Is it possible to compare PSA recurrence-free survival after surgery and radiotherapy using revised ASTRO criterion-"nadir + 2"? Urology 72: 389-393discussion 394-5.

Parikh H, Wang Z, Pettigrew KA, Jia J, Daugherty S, Yeager M, Jacobs KB, Hutchinson A, Burdett L, Cullen M, Qi L, Boland J, Collins I, Albert TJ, Vatten LJ, Hveem K, Njolstad I, Cancel-Tassin G, Cussenot O, Valeri A, Virtamo J, Thun MJ, Feigelson HS, Diver WR, Chatterjee N, Thomas G, Albanes D, Chanock SJ, Hunter DJ, Hoover R, Hayes RB, Berndt SI, Sampson J, Amundadottir L (2011) Fine mapping the KLK3 locus on chromosome 19q13.33 associated with prostate cancer susceptibility and PSA levels. Hum Genet 129: 675-685.

Penney KL, Salinas CA, Pomerantz M, Schumacher FR, Beckwith CA, Lee GS, Oh WK, Sartor O, Ostrander EA, Kurth T, Ma J, Mucci L, Stanford JL, Kantoff PW, Hunter DJ, Stampfer MJ, Freedman ML (2009) Evaluation of 8q24 and 17q risk loci and prostate cancer mortality. Clin Cancer Res 15: 3223-3230.

Penney KL, Schumacher FR, Kraft P, Mucci LA, Sesso HD, Ma J, Niu Y, Cheong JK, Hunter DJ, Stampfer MJ, Hsu SI (2011) Association of KLK3 (PSA) genetic variants with prostate cancer risk and PSA levels. Carcinogenesis 32: 853-859.
Pomerantz MM, Werner L, Xie W, Regan MM, Lee GS, Sun T, Evan C, Petrozziello G, Nakabayashi M, Oh WK, Kantoff PW, Freedman ML (2011) Association of prostate cancer risk Loci with disease aggressiveness and prostate cancer-specific mortality. Cancer Prev Res (Phila) 4: 719-728.

Schumacher FR, Berndt SI, Siddiq A, Jacobs KB, Wang Z, Lindstrom S, Stevens VL, Chen C, Mondul AM, Travis RC, Stram DO, Eeles RA, Easton DF, Giles G, Hopper JL, Neal DE, Hamdy FC, Donovan JL, Muir K, Al Olama AA, Kote-Jarai Z, Guy M, Severi G, Gronberg H, Isaacs WB, Karlsson R, Wiklund F, Xu J, Allen NE, Andriole GL, Barricarte A, Boeing H, Bueno-de-Mesquita HB, Crawford ED, Diver WR, Gonzalez CA, Gaziano JM, Giovannucci EL, Johansson M, Le Marchand L, Ma J, Sieri S, Stattin P, Stampfer MJ, Tjonneland A, Vineis P, Virtamo J, Vogel U, Weinstein SJ, Yeager M, Thun MJ, Kolonel LN, Henderson BE, Albanes D, Hayes RB, Feigelson HS, Riboli E, Hunter DJ, Chanock SJ, Haiman CA, Kraft P (2011) Genome-wide association study identifies new prostate cancer susceptibility loci. Hum Mol Genet 20: 3867-3875.

Shui IM, Lindstrom S, Kibel AS, Berndt SI, Campa D, Gerke T, Penney KL, Albanes D, Berg C, Bueno-De-Mesquita HB, Chanock S, Crawford ED, Diver WR, Gapstur SM, Gaziano JM, Giles GG, Henderson B, Hoover R, Johansson M, Le Marchand L, Ma J, Navarro C, Overvad K, Schumacher FR, Severi G, Siddiq A, Stampfer M, Stevens VL, Travis RC, Trichopoulos D, Vineis P, Mucci LA, Yeager M, Giovannucci E, Kraft P (2014) Prostate cancer (PCa) risk variants and risk of fatal PCa in the National Cancer Institute Breast and Prostate Cancer Cohort Consortium. Eur Urol 65: 1069-1075.

Stephenson AJ, Kattan MW, Eastham JA, Bianco Jr FJ, Yossepowitch O, Vickers AJ, Klein EA, Wood DP, Scardino PT (2009) Prostate cancerspecific mortality after radical prostatectomy for patients treated in the prostate-specific antigen era. J Clin Oncol 27: 4300-4305.

Stephenson AJ, Kattan MW, Eastham JA, Dotan ZA, Bianco Jr FJ, Lilja H, Scardino PT (2006) Defining biochemical recurrence of prostate cancer after radical prostatectomy: a proposal for a standardized definition. J Clin Oncol 24: 3973-3978.

Szulkin R, Holmberg E, Stattin P, Xu J, Zheng S, Palmgren J, Gronberg H, Wiklund F (2012) Prostate cancer risk variants are not associated with disease progression. Prostate 72: 30-39.

Takata R, Akamatsu S, Kubo M, Takahashi A, Hosono N, Kawaguchi T, Tsunoda T, Inazawa J, Kamatani N, Ogawa O, Fujioka T, Nakamura Y, Nakagawa H (2010) Genome-wide association study identifies five new susceptibility loci for prostate cancer in the Japanese population. Nat Genet 42: 751-754.

Whitaker HC, Kote-Jarai Z, Ross-Adams H, Warren AY, Burge J, George A, Bancroft E, Jhavar S, Leongamornlert D, Tymrakiewicz M, Saunders E, Page E, Mitra A, Mitchell G, Lindeman GJ, Evans DG, Blanco I, Mercer C, Rubinstein WS, Clowes V, Douglas F, Hodgson S, Walker L, Donaldson A, Izatt L, Dorkins H, Male A, Tucker K, Stapleton A, Lam J, Kirk J, Lilja H, Easton D. Committee I S S. Collaborators I S Collaborators U G. Cooper C, Eeles R, Neal DE (2010) The rs10993994 risk allele for prostate cancer results in clinically relevant changes in microseminoprotein-beta expression in tissue and urine. PLoS One 5: e13363.

Wiklund FE, Adami HO, Zheng SL, Stattin P, Isaacs WB, Gronberg H, Xu J (2009) Established prostate cancer susceptibility variants are not associated with disease outcome. Cancer Epidemiol Biomarkers Prev 18: $1659-1662$.

Willis JA, Olson SH, Orlow I, Mukherjee S, Mcwilliams RR, Kurtz RC, Klein RJ (2012) A replication study and genome-wide scan of single-nucleotide polymorphisms associated with pancreatic cancer risk and overall survival. Clin Cancer Res 18: 3942-3951.

Xu X, Valtonen-Andre C, Savblom C, Hallden C, Lilja H, Klein RJ (2010) Polymorphisms at the Microseminoprotein-beta locus associated with physiologic variation in beta-microseminoprotein and prostate-specific antigen levels. Cancer Epidemiol Biomarkers Prev 19: 2035-2042.

This work is published under the standard license to publish agreement. After 12 months the work will become freely available and the license terms will switch to a Creative Commons AttributionNonCommercial-Share Alike 4.0 Unported License 